\title{
Bases Neurais da Ansiedade Matemática: implicações para o processo de ensino-aprendizagem
}

\author{
Neural Bases of Mathematical Anxiety - implications for the teaching-learning \\ process
}

\author{
Marcos Guilherme Moura-Silva* \\ ORCID iD 0000-0003-3589-1897 \\ João Bento Torres Neto** \\ ORCID iD 0000-0002-9155-9445
}

Tadeu Oliver Gonçalves ${ }^{* * *}$

ORCID iD 0000-0002-2704-5853

\begin{abstract}
Resumo
A Ansiedade Matemática é um fenômeno global e altamente prevalente, possuindo marcadores fisiológicos, cognitivos e comportamentais. No entanto, pouco se conhece sobre seus mecanismos neurais subjacentes. Fornecemos uma Revisão Sistemática de estudos que investigaram os correlatos neurais da Ansiedade Matemática (AM) na última década e discutimos suas implicações para o processo de ensino aprendizagem. Foram selecionadas pesquisas que avaliaram parâmetros fisiológicos da função cerebral de indivíduos com AM através de bancos de dados eletrônicos, atentando-se a critérios de inclusão e exclusão delineados. A qualidade da literatura foi analisada a partir dos 11 itens da escala de qualidade PEDro e conduzida pelo fluxograma de seleção de estudos PRISMA, resultando na inclusão de 14 estudos neurocientíficos. Em geral, a literatura vem sugerir que as redes neurais de medo e de dor são estimuladas antes e durante tarefas numéricas em indivíduos com alta AM. Além disso, há uma capacidade reduzida de Memória de Trabalho e déficit de atenção/inibição em indivíduos com alta AM. Eles também são mais propensos a cometer erros em tarefas matemáticas, tem representações menos precisas de magnitude numérica, abordam os problemas matemáticos de maneira diferente de seus pares menos ansiosos e tendem a elevar mais recursos de controle cognitivo para concluir objetivos com estímulos aversivos relacionados ao raciocínio matemático, podendo impactar a eficiência de processamento e gerar déficits de desempenho. Resultados suportam, ainda, que os efeitos da AM estão associados à uma menor ativação cortical já durante os estágios iniciais do

\footnotetext{
* Doutor em Educação em Ciências e Matemáticas pela Universidade Federal do Pará (UFPA). Professor Adjunto pela Universidade Federal do Pará (UFPA), Belém, Pará, Brasil. Endereço para correspondência: Universidade Federal do Pará, Rua Augusto Corrêa, 1, Guamá, Belém, Pará, Brasil, CEP: 66075-110. E-mail: marcosgmouras@yahoo.com.br.

** Doutor em Neurociências e Biologia Celular pela Universidade Federal do Pará (UFPA) com período sanduíche na University of Western Ontário, Canada. Pós-doutorado no Brain Aging \& Cognitive Health Lab da universidade de Pittsburgh. Professor adjunto na Univesidade Federal do Pará (UFPA), Belém, Pará, Brasil. Endereço para correspondência: Universidade Federal do Pará, Rua Augusto Corrêa, 1, Guamá, Belém, Pará, Brasil, CEP: 66075110. E-mail: bentotorres@gmail.com

*** Doutor em Educação Matemática pela Universidade Estadual de Campinas (UNICAMP). Professor Titular pela Universidade Federal do Pará (UFPA), Belém, Pará, Brasil. Endereço: Universidade Federal do Pará, Rua Augusto Corrêa, 1, Guamá, Belém, Pará, Brasil, CEP: 66075-110. E-mail: tadeuoliver@yahoo.com.br
} 
processamento de estímulos numéricos, independente da complexidade da tarefa. Implicações para guiar a prática do professor que ensina Matemática são discutidas à luz das evidências.

Palavras-chave: Ansiedade matemática. Bases Neurais. Revisão Sistemática. Ensino aprendizagem.

\begin{abstract}
Mathematical Anxiety is a global phenomenon and highly prevalent, possessing physiological, cognitive, and behavioral markers. However, little is known about its underlying neural mechanisms. We provide a Systematic Review of studies that have investigated the neural correlates of Mathematical Anxiety in the last decade and discuss its implications for the teaching-learning process. We selected studies that made brain measurements of individuals with Mathematical Anxiety through electronic databases, considering the delineated inclusion and exclusion criteria. The quality of the literature was analyzed from the 11 items of the PEDro quality scale and conducted by the selection flowchart of PRISMA studies, resulting in the inclusion of 13 neuroscientific studies. In general, literature suggests that neural bases of fear and pain are stimulated by Mathematical Anxiety before and during numerical tasks. There is a reduced capacity for Working Memory and Attention Deficit / Inhibition in individuals with high Mathematical Anxiety. They are also more likely to make mistakes in mathematical tasks, have less precise representations of numerical magnitude, approach math problems differently from their less anxious peers, and tend to raise more cognitive control resources to complete goals with aversive stimuli, processing efficiency and generating performance deficits. Results also support that the effects of Mathematical Anxiety are associated with less cortical activation during the initial stages of numerical stimuli processing, regardless of task complexity.
\end{abstract}

Keywords: Mathematical Anxiety. Neural Bases. Systematic Review. Teaching learning.

\title{
1 Ansiedade matemática
}

Estudos envolvendo Ansiedade Matemática (doravante, AM) vem recebendo atenção crescente nos últimos anos, desde que o conceito de "ansiedade a números" foi introduzido por Dreger e Aiken em relatório publicado em 1957. Neste relatório, os autores propuseram três assertivas: a) que a AM difere da ansiedade geral, embora uma se sobreponha a outra em certo grau; b) que a AM não estaria diretamente ligada à inteligência geral; c) que haveria uma relação inversa entre AM e desempenho acadêmico em cursos de Matemática (ver ASHCRAFT; MOORE, 2009). Pesquisas subsequentes confirmaram repetidamente todas essas previsões, como destacado nas metanálises de Hembree (1990), Dowker et al., (2016), Suaréz-Pellicioni et al. (2016) e Carey et al. (2016).

A AM é um fenômeno global e altamente prevalente. Estima-se que uma parcela significativa da população experimente medo e apreensão quando confrontada com problemas numéricos (HEMBREE, 1990; ASHCRAFT, 2002; HLALELE, 2012; MUTODI; NGIRANDE, 2014, JANSEN et al., 2013). No estudo do PISA (Programme for International Student Assessment), em 2012, do qual participaram alunos de 65 países, quase $60 \%$ dos estudantes 
relataram preocupações relativas as aulas de Matemática e 30\% disseram se sentir impotentes ao fazerem um problema matemático (OECD, 2013).

A AM possui marcadores fisiológicos, como, por exemplo, ativação de áreas cerebrais relacionadas à percepção de dor (LYONS; BEILOCK, 2012a); marcadores cognitivos, como as limitações de Memória de Trabalho durante tarefas matemáticas complexas (ASHCRAFT; KIRK, 2001; ASHCRAFT; RIDLEY, 2005); marcadores comportamentais, a exemplo da negação de carreiras que enfatizem habilidades numéricas por conta de pobre desempenho (HEMBREE, 1990; BROWN et al., 2008) ou na tomada de decisões envolvendo itens de consumo (JONES et al., 2012). A AM se conceitua como uma "sensação de tensão e ansiedade que interfere na manipulação dos números e na resolução de problemas matemáticos, relacionados a uma grande variedade de situações da vida ordinária e acadêmica" (RICHARDSON; SUINN, 1972, p.551; ASHCRAFT; FAUST, 1994).

Em síntese, a AM é um fenômeno que tem um impacto considerável no desempenho de tarefas matemáticas (ver ASHCRAFT; KIRK, 2001). Dados neurocientíficos podem proporcionar melhores perspectivas de intervenções e guiar a prática do professor que ensina Matemática. Considerando que, na última década, distintos estudos forneceram evidências que possibilitaram perscrutar as bases neurais ${ }^{1}$ da $\mathrm{AM}$, fornecemos uma Revisão Sistemática da literatura recente com o objetivo de apresentar estudos que investigaram os correlatos neurais de indivíduos com Ansiedade Matemática, destacando implicações para o processo de ensino e aprendizagem.

\section{Método}

As bases de dados eletrônicas PubMed e ERIC foram eleitas para o processo de busca de estudos que investigaram os correlatos neurais da Ansiedade Matemática. Termos-chave de busca incluíram a combinação das palavras "Brain, Brain Bases, Anxiety Mathematical, Arithmetic", dentre outras palavras equivalentes.

Para essa Revisão Sistemática, incluiu-se todos os estudos que: i) investigaram as bases neurais da Ansiedade Matemática; ii) foram escritos em língua inglesa; iii) publicados entre 2008 e 2018; iv) realizados com escolares ou não escolares, independente da idade; v) envolveram tarefas numéricas e vi) apresentaram implicações diretas para o processo de ensino e

1 Processos e mecanismos fisiológicos do sistema nervoso central envolvidos em determinada atividade, comportamento ou função. 
aprendizagem. Foram excluídos estudos que: i) foram desenvolvidos com populações especiais (por exemplo, alunos com discalculia); ii) não apresentaram implicações educacionais; iv) investigaram bases neurais vinculadas a intervenções de medicação da Ansiedade Matemática.

A qualidade dos estudos elegidos foi avaliada de modo independente pelo primeiro autor, baseado nos 11 itens da escala do Banco de Dados de Evidências Fisioterapêuticas (PEDro), conforme Quadro 1. A referida escala, amplamente utilizada na avaliação de metanálises, afere de forma confiável características dos estudos, como randomização, cegamento, comparação entre grupos e medidas de variabilidade (SHIWA et al., 2011).

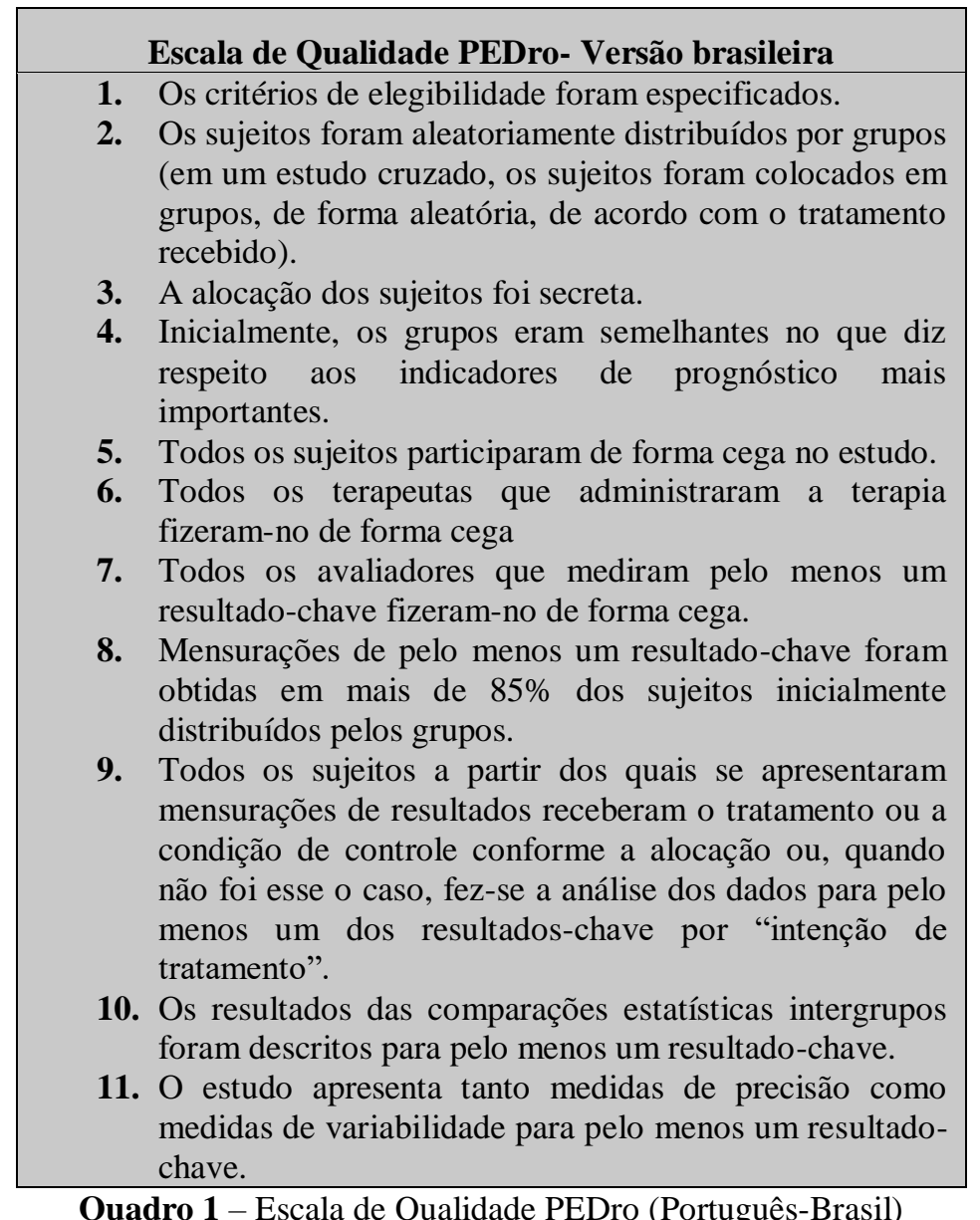

Quadro 1 - Escala de Qualidade PEDro (Português-Brasil)

Fonte: SHIWA et al. (2011)

O fluxograma PRISMA foi utilizado como parâmetro para conduzir a identificação, triagem, elegibilidade e inclusão de estudos para essa Revisão Sistemática, conforme Figura 1. 

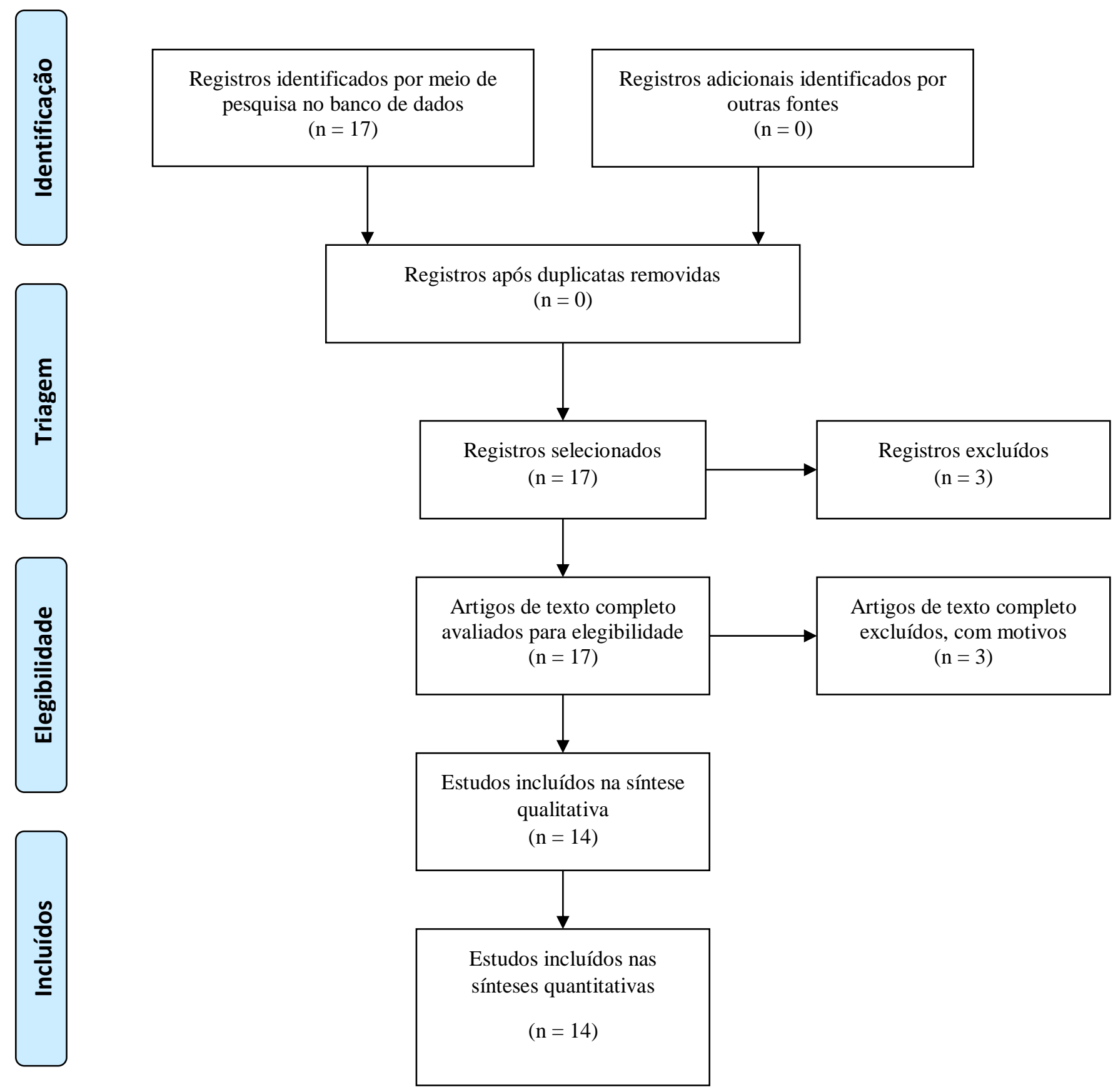

Figura 1 - Fluxograma de seleção de estudos PRISMA Fonte: MOHER et al. (2009).

Dos 17 estudos identificados, 3 foram excluídos por não atenderem aos critérios de inclusão. Um deles, o estudo de Jones et al. (2012), investigou como o cérebro de indivíduos com alta AM muda quando tem que decidir pela compra de itens de consumo, não apresentando 
implicações diretas para o processo de ensino e aprendizagem. O estudo de Supekar et al. (2015), que investigou os correlatos neurais após a intervenção de uma intensa tutoria cognitiva e o estudo de Sarkar et al. (2014) que investigou uma intervenção fazendo uso de Estimulação Transcraniana por Corrente Contínua (ETCC) ${ }^{2}$ também foram desconsiderados para o escopo de análise desse estudo. Análises pormenorizadas dos artigos incluídos serão discutidas a seguir.

\section{Bases neurais da Ansiedade Matemática}

A partir dos resultados recentes de estudos que fizeram uso de fRMI (Imagem por Ressonância Magnética Funcional ${ }^{3}$ ) ou medidas de reatividade eletrofisiológica, como Potenciais de Eventos Relacionados'- PER (em inglês, ERP) /Eletroencefalograma- EEG, foi possível perscrutar as principais regiões cerebrais relacionadas à Ansiedade Matemática. Trata-se de esforços ainda incipientes, mas que corroboram, aprimoram e avançam os resultados dos estudos psicológicos conduzidos ao longo dos últimos 60 anos. As evidências apontam que, pelo menos, duas redes estão relacionadas à "emotividade" da Ansiedade Matemática no cérebro: a rede de dor, abrangendo a ínsula (LYONS; BEILOCK, 2012a; LYONS; BEILOCK, 2012b) e a rede de medo, envolvendo a amígdala (YOUNG et al., 2012.

Em relação à primeira, rede de dor, os estudos conduzidos por Lyons e Beilock (2012b) trouxeram evidências que explicam a natureza subjetiva da Ansiedade Matemática. Enquanto as atividades cerebrais eram medidas por fMRI, quatorze participantes com alta AM e quatorze indivíduos com baixa AM tiveram que responder, intercaladamente, blocos de tarefas matemáticas e de palavras. Um círculo amarelo ou um quadrado azul indicavam antecipadamente se a atividade envolveria cálculo ou vocábulo.

\footnotetext{
${ }^{2}$ Trata-se da utilização de corrente elétrica baixa e contínua emitida diretamente na área cerebral de interesse através de pequenos eletrodos fixados no couro cabeludo.

${ }^{3}$ A Ressonância Magnética Funcional é uma técnica específica que se utiliza de fortes ondas magnéticas capazes de detectar variações do fluxo sanguíneo cerebral em resposta à atividade neural.

${ }^{4}$ Potenciais de Eventos relacionados é uma análise que permite identificar atividade cerebral quando o indivíduo é estimulado, seja interna ou externamente. Essa análise é mensurada por eletroencefalograma (EEG), que, por sua vez, consiste em uma técnica de monitoramento das atividades elétricas do cérebro através de eletrodos colocados no couro cabeludo.
} 
Notou-se que, ao se antecipar uma tarefa de Matemática, mais aumentava a atividade em regiões associadas às ameaças viscerais (ínsula dorso-posterior bilateral) nos indivíduos AM (Figura 2), experimentando-se muitas das vezes a experiência da própria dor (LAMM et al., 2011); fato não constatado em indivíduos com baixa AM. Lyons e Beilock (2012b, p.1) sugerem que "a ativação da rede de dor está subjacente ao fato de que simplesmente antecipar um evento temido, pode ser doloroso".

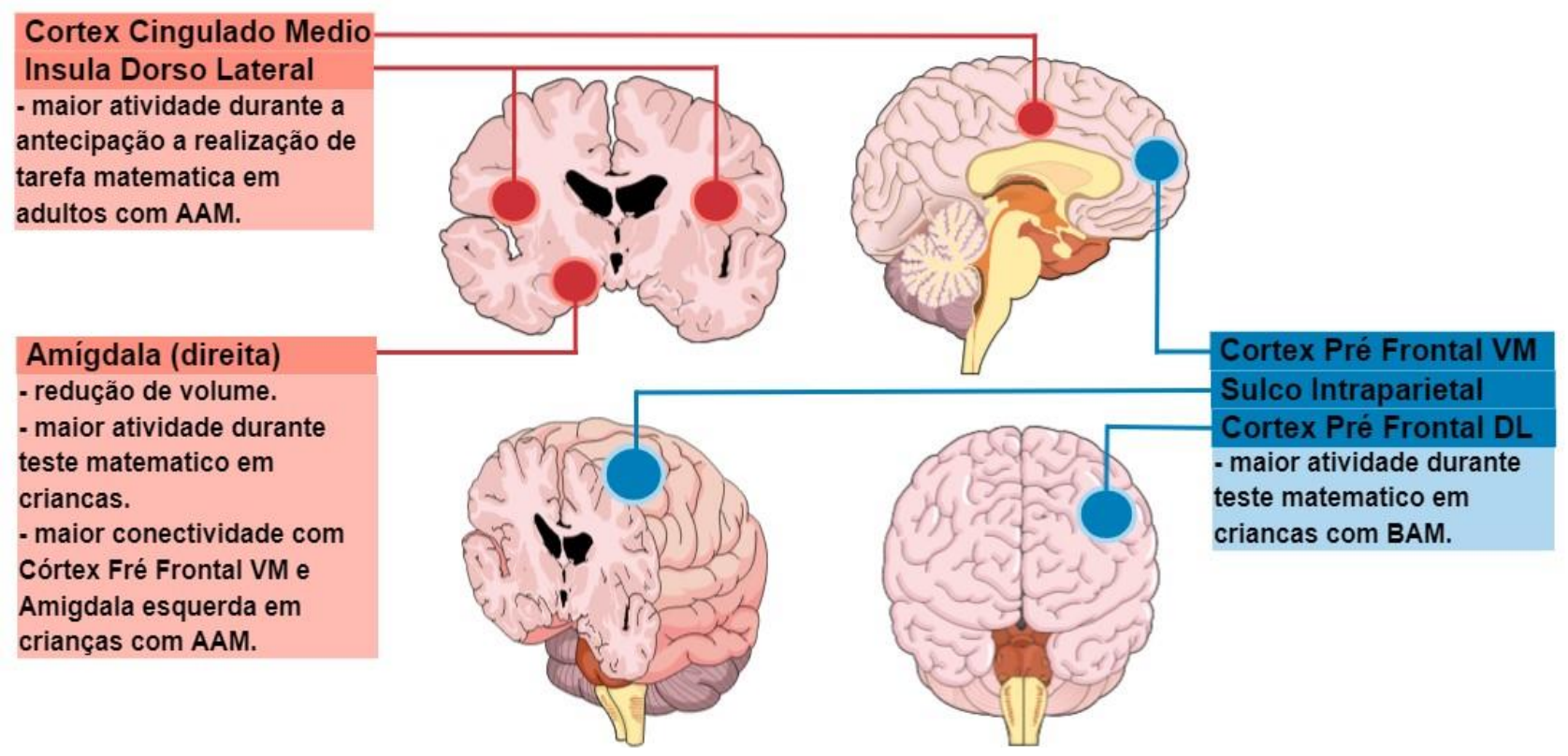

Figura 2 - Síntese dos principais achados em estudos de imagem por ressonância magnética funcional Fonte: Adaptado de Lyons e Beilock (2012) Young et al. (2012) e Kucian et al. (2018)

Em vermelho estão representadas as estruturas que apresentam aumento de atividade quando sujeitos com alta ansiedade matemática (AAM) estão prestes a fazer ou fazendo atividades matemáticas. Em azul são representadas as áreas cerebrais mais ativadas em crianças com baixa ansiedade matemática (BAM). Note que a AAM ativa mais regiões que compões as redes neurais de medo e dor (vermelho) quando deveriam ser mais ativadas as regiões associadas com o processamento matemático (proximidades do Sulco Intraparietal), de resolução de problemas e controle executivo como o Córtices pré-frontais Ventromedial (VM) e Dorsolateral (DL) (azul).

Se a experiência da AM gera uma reação corporal visceral aversiva, tais resultados fornecem evidências significativas quanto ao potencial mecanismo neural que explica por que pessoas que apresentam alta AM tendem a evitar situações relacionadas à disciplina, seja em seu cotidiano, seja em sala de aula ou até mesmo em suas trajetórias acadêmicas.

Os achados de Lyons e Beilock (2012a) também fornecem explicações do porquê indivíduos com mesmo grau de AM, apresentam desempenhos matemáticos variados. Por encontrarem atividades em regiões frontais do cérebro, envolvidas na regulação de emoções negativas, os autores partiram do pressuposto de que a redução de déficits matemáticos seria 
resultado do aumento do controle cognitivo, que permitiria aos participantes ansiosos ir mudando o desempenho ao longo da resolução de tarefas matemáticas.

Em outros termos, poder-se-ia perguntar até que ponto os indivíduos com alta AM conseguiriam recrutar áreas cerebrais relacionadas ao controle atencional e regulação de emoções? A hipótese era que, quanto maior a atividade no córtex pré-frontal dorsolateral, região envolvida em funções executivas como atenção, inibição e memória de trabalho (BRASS et al., 2005), mais os indivíduos com alta AM estariam no controle de resposta negativa, e, portanto, conseguiriam obter um melhor desempenho. Essa hipótese foi confirmada, concluindo que o desempenho matemático de indivíduos ansiosos está associado à capacidade de acelerar recursos de controle atencional.

A esse respeito, o estudo conduzido por Sarkar et al. (2014) fez uso de Estimulação Transcraniana de Corrente Contínua (ETCC), emitida justamente no córtex pré-frontal dorsolateral, um alvo frequente para a modulação da regulação emocional. Os autores relataram melhoras no tempo de resposta para problemas aritméticos simples e uma diminuição nas concentrações de cortisol (um biomarcador de estresse), em indivíduos com alta AM, embora tenha se percebido efeito contrário em estudantes com baixa AM. Segundo os autores, os diferentes níveis de AM produzem estados neurais diferentes que são específicos da tarefa e da situação. Nesse sentido, essas distintas assinaturas neurais ao interagirem com a ETCC produzem resultados diferentes, influenciando os efeitos de estimulação (SARKAR et al., 2014).

Criticamente, as evidências de Klados et al. (2017) foram consistentes com os achados de Lyons e Beilock (2012a) ao perceberem que a ativação de redes cerebrais em indivíduos ansiosos muda durante a fase de antecipação de uma experiência relacionada à Matemática. No estudo de Klados et al. (2017), notou-se atividade em todas as bandas de frequência ${ }^{5}$ do EEG em contraste com os pares menos ansiosos. Antecipando uma próxima tarefa matemática, regiões associadas à dor foram ativadas na ínsula dorso posterior bilateral, o que viria explicar as ativações nas bandas de frequência beta e gama, segundo os autores. A frequência na banda theta, por sua vez, foi avaliada como resposta as expressões emocionais ou a alta demanda de recursos na Memória de Trabalho para controlar emoções negativas, em contraposição aos menos ansiosos, onde a

\footnotetext{
5 Bandas de frequência se referem às frequências de sinais da atividade cerebral medida por eletroencefalograma. Elas são quatro: Delta - tem uma frequência menor ou igual à $3 \mathrm{~Hz}$; Theta - tem frequência entre $3,5 \mathrm{a} 7,5 \mathrm{~Hz}$ e é classificada como atividade lenta; Alfa - frequência entre 7,5 a $13 \mathrm{~Hz}$; e Beta - tem uma frequência igual ou maior à $14 \mathrm{~Hz}$, sendo classificada como atividade rápida.
} 
Memória de Trabalho se mostra mais eficiente (ver KLADOS et al., 2017). Esses achados estão em consonância com os de Pletzer et al. (2015), no qual jovens adultos com alta AM apresentavam retardo na desativação na rede de modo padrão em momentos que precediam a realização da tarefa matemática, evidenciando redução na eficiência de processamento decorrente da necessidade e dispensa de novos esforços para controle das emoções negativas associadas à manipulação numérica.

Lyons e Beilock (2012b) sugerem que intervenções educativas que enfatizam o controle de fatores emocionais negativos tendem a ser mais eficazes para promover uma população matematicamente proficiente, quando comparadas com interferências que foquem meramente em treino adicional de Matemática.

Em relação à segunda, rede de medo, Young et al. (2012), notaram uma conectividade aberrante da amígdala direita (que são importantes no processamento de emoções negativas) em crianças de 7 a 9 anos com AM (figura 2). Considerando que a amígdala é conhecida pela percepção do medo (PHELPS; LEDOUX, 2005), sua ativação durante uma tarefa matemática vem confirmar o medo da Matemática das crianças. Ao mesmo tempo, perceberam uma associação da AM com resposta reduzida nas regiões do córtex pré-frontal dorsolateral e parietal posterior (Figura 2), envolvidas na memória de trabalho e no raciocínio matemático (MENON et al., 2000; WENDELKEN, 2015). Esses resultados vão ao encontro de estudos anteriores que pensaram a influência da AM sobre o desempenho a partir da capacidade reduzida da memória de trabalho (como por exemplo, ASHCRAFT; KIRK, 2001; BEILOCK; DECARO, 2007).

Além disso, a conectividade da amígdala com outras áreas cerebrais também foi observada por Young et al. (2012). Enquanto em crianças com baixa AM, a amígdala foi acoplada às áreas cerebrais que facilitam o processamento eficiente de tarefas, em crianças com alta ansiedade, a amígdala mostrou maior conectividade com regiões envolvidas no processamento e regulação de emoções negativas (uma espécie de mecanismo compensatório de desempenho) (YOUNG et al., 2012). Há evidências de que a amígdala está envolvida em comportamentos cognitivo-emocionais complexos a partir de suas interações com distintas áreas cerebrais (PESSOA, 2008; LEDOUX; PHELPS, 2008).

O estudo conduzido por Pizzie e Kraemer (2017) confirma essa ativação da amígdala em resposta a uma breve exposição a estímulos matemáticos, ampliando os resultados para adultos jovens. Esses dados vêm ilustrar que, mesmo sem a expectativa ou o ato de resolver problemas, 
existe uma resposta neural relacionada à ameaça e vigilância por parte dos indivíduos com AM, semelhante a fobias específicas (como medo de cobra, por exemplo).

Outra implicação importante do estudo de Pizzie e Kraemer (2017) é mostrar que essa responsividade da amígdala durante os ensaios envolvendo os estímulos matemáticos, parecem ser característica específica da Ansiedade Matemática, pois não foi observado o mesmo comportamento em relação à ansiedade-traço. Esses resultados se apresentam consistentes com os obtidos em pesquisas anteriores (como YOUNG et al., 2012; LYONS; BEILOCK, 2012a).

\begin{tabular}{|c|c|c|c|c|}
\hline Referência & População & Procedimentos & Correlatos neurais & Implicações \\
\hline $\begin{array}{l}\text { Lyons e Beilock } \\
\text { (2012a) }\end{array}$ & $\begin{array}{c}28 \\
\text { participantes } \\
\text { adultos }\end{array}$ & $\begin{array}{l}\text { Os sujeitos realizaram } 2 \\
\text { tarefas distintas } \\
\text { (matemática e tarefa de } \\
\text { palavras) em dois níveis de } \\
\text { dificuldade para cada } \\
\text { tarefa: difícil e fácil. Antes } \\
\text { de cada bloco de ensaio, } \\
\text { foi fornecida uma sugestão } \\
\text { que indicava o tipo de } \\
\text { tarefa que estava prestes a } \\
\text { seguir. A atividade neural } \\
\text { foi medida por fMRI. }\end{array}$ & $\begin{array}{l}\text { Regiões frontoparietais } \\
\text { inferiores foram associadas a } \\
\text { indivíduos com AM. A } \\
\text { atividade nessas regiões está } \\
\text { associada ao controle } \\
\text { cognitivo de processos de } \\
\text { alto nível, } \\
\text { deslocamento de tarefa e } \\
\text { inibição de respostas } \\
\text { (BRASS et al. 2005; } \\
\text { DERRFUSS et al. 2005, } \\
\text { 2009). }\end{array}$ & $\begin{array}{l}\text { Os déficits de desempenho } \\
\text { matemático exibidos por } \\
\text { alguns indivíduos com alta } \\
\text { AM podem ser consequência } \\
\text { da elevação de recursos de } \\
\text { controle cognitivo por parte } \\
\text { desses indivíduos ao } \\
\text { antecipar uma tarefa } \\
\text { matemática. Os resultados } \\
\text { sugerem, portanto, que as } \\
\text { melhores práticas para } \\
\text { melhorar a competência } \\
\text { matemática de quem tem AM } \\
\text { não é gerar mais cursos de } \\
\text { Matemática. Ao invés disso, } \\
\text { focar em práticas de sala de } \\
\text { aula que ajudem os alunos a } \\
\text { organizar recursos de } \\
\text { controle cognitivo. }\end{array}$ \\
\hline $\begin{array}{l}\text { Lyons e Beilock } \\
\text { (2012b) }\end{array}$ & $\begin{array}{c}28 \\
\text { participantes } \\
\text { adultos }\end{array}$ & $\begin{array}{l}\text { Os } \\
\text { completaram uma tarefa de } \\
\text { palavra e uma tarefa } \\
\text { matemática (desenho de } \\
\text { bloco) enquanto } r \\
\text { atividade neural foi } \\
\text { medida, usando fMRI. }\end{array}$ & $\begin{array}{l}\text { A ínsula dorso-posterior } \\
\text { (INSP) e o Córtex Médio- } \\
\text { Cingulado (MCC) mostraram } \\
\text { maior conectividade ao se } \\
\text { antecipar uma tarefa de } \\
\text { matemática. Essas regiões } \\
\text { estão implicadas na } \\
\text { percepção da dor visceral } \\
\text { (advindas de estímulos } \\
\text { ameaçadores). }\end{array}$ & $\begin{array}{l}\text { Se a experiência de } \\
\text { Ansiedade Matemática se } \\
\text { baseia em uma reação } \\
\text { corporal visceral e aversiva } \\
\text { (relacionada à percepção de } \\
\text { dor), pode-se explicar por } \\
\text { que indivíduos com Alta AM } \\
\text { evitam situações e problemas } \\
\text { relacionados à matemática. }\end{array}$ \\
\hline
\end{tabular}




\section{Young et al. (2012) \\ Suaréz Pellicioni et al. (2013a)

46 crianças
entre 7-9
anos anos \\ As crianças verificaram problemas simples e envolvendo subtração, informando se estavam corretos ou incorretos, enquanto eram monitoradas por fMRI. \\ 26 estudantes universitários \\ Os participantes \\ completaram tarefas de verificação de sentenças do tipo $\mathrm{a}+\mathrm{b}=\mathrm{c}$, algumas com soluções extremamentes incorretas, enquanto eram monitorados usando medidas de reatividade eletrofisiológica- PER (Potenciais de Eventos Relacionados)}

A AM foi associada à hiperatividade anormal da amígdala, uma região do cérebro associada ao processamento de emoções negativas e estímulos de medo (PHELPS; LEDOUX, 2005). Em crianças com alta AM, foram encontradas respostas reduzidas em áreas corticais e subcorticais que têm sido consistentemente associadas ao raciocínio matemático em crianças e adultos (MENON; RIVERA; WHITE; GLOVER; REISS, 2000). Além disso, crianças ansiosas mostraram respostas reduzidas em regiões envolvidas na memória de trabalho e atenção, incluindo o córtex pré-frontal dorsolateral, área motora suplementar e núcleos da base (CHANG; CROTTAZHERBETTE; MENON, 2007).

Foi encontrado um componente $\mathrm{P} 600 / \mathrm{P} 3 \mathrm{~b}$ de maior amplitude e atraso de latência para o grupo de alta AM em comparação com os pares de baixa AM. Contudo, isso foi observado apenas para as soluções extremamente incorretas.
Suaréz Pellicioni et al. (2013b)

\section{4} participantes adultos
Os participantes resolveram tarefas Stroop numéricas e tarefas Stroop Clássicas enquanto eram monitorados usando medidas de reatividade eletrofisiológicaPER (Potenciais de Eventos Relacionados)
Foi encontrado o componente de Negatividade de Erro Relacionado (ERN) no grupo dos indivíduos com alta AM quando estes cometeram erros na tarefa numérica de Stroop, mas não na tarefa clássica de Stroop.
Essas evidências suportam o fato de que a AM se desenvolve em crianças e destacam a importância de avaliá-la e tratá-la precocemente.

Necessariamente, a AM influencia o desempenho a partir da capacidade reduzida da Memória de Trabalho, da atenção e de processos de controle cognitivo durante a resolução de problemas matemáticos.

Considerando que a amplitude deste componente indica a quantidade de recursos de atenção atribuídos a um estímulo; e sua latência reflete a velocidade para processar estímulos, pode-se concluir que indivíduos ansiosos estavam gastando mais tempo e recursos no processamento de uma solução que poderia prontamente ser ignorada dada a obviedade de seu erro. Isso implica em um déficit de atenção/inibição em indivíduos com alta AM. Os dados sugerem que indivíduos com alta $\mathrm{AM}$ são mais propensos a autogerar erros em tarefas numéricas. Considerando que os erros são vistos de modo negativo em tarefas matemáticas, provavelmente ele constitui fator contributivo no desenvolvimento manutenção da AM 


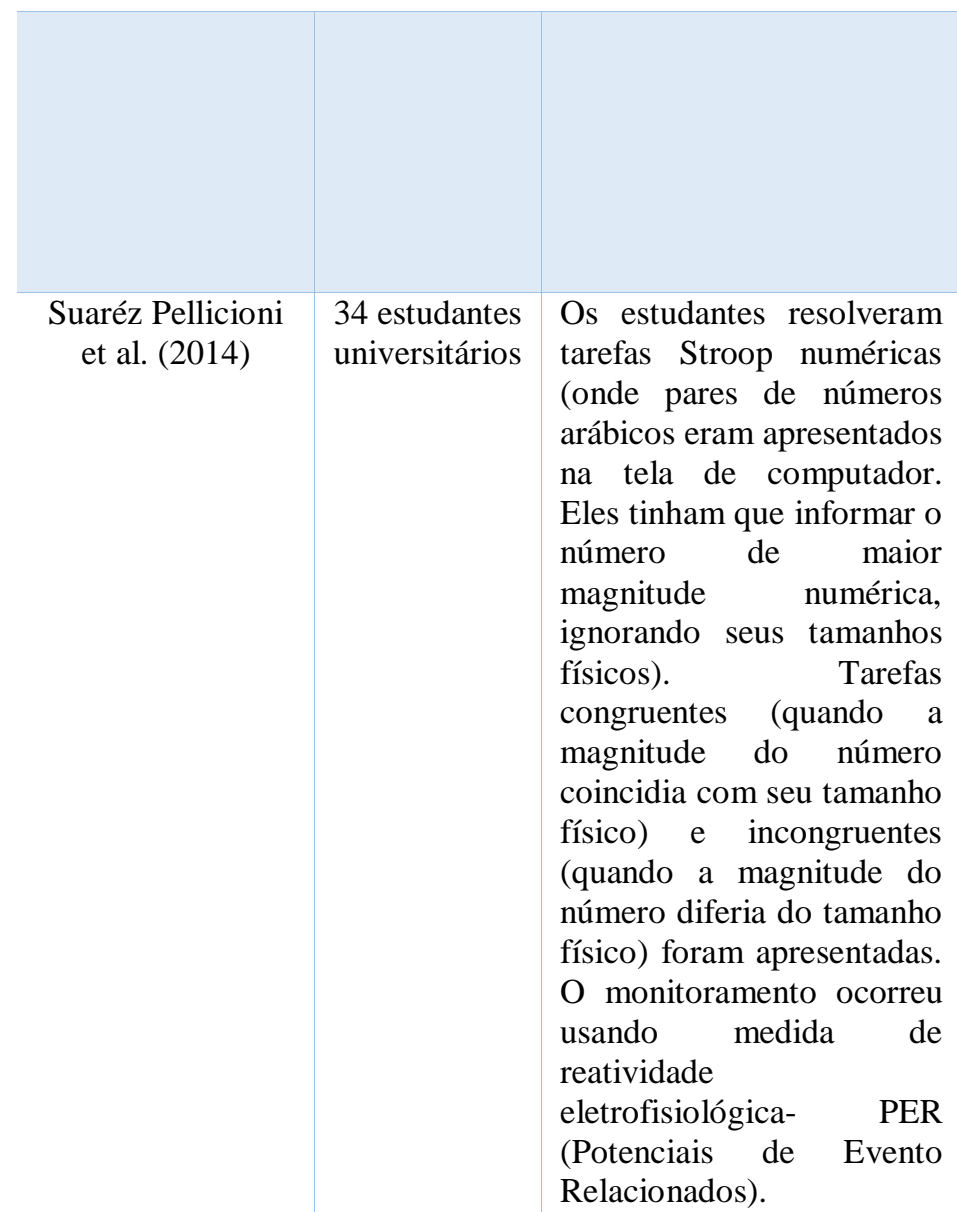

Núñes Peña e Suaréz Pellicioni (2014) 53 participantes adultos

Núnes-Peña e 34 estudantes Suaréz Pellicioni universitários (2015)
Os participantes foram apresentados a pares de algarismos indo-arábicos e solicitados a decidir qual deles apresentavam maior magnitude numérica, enquanto eram monitorados pela medida de reatividade eletrofisiológica- PER (Potencias de Eventos Relacionados)

Os estudantes foram solicitados a calcular e verificar se equações envolvendo adições de dois dígitos eram verdadeiras ou falsas,

O componente N450, que está associado ao processamento de conflitos, foi observado em indivíduos com alta ansiedade Matemática a 
enquanto eram estímulos que provocam monitorados por sensores sentimentos negativos, sendo de EEG. considerada um indicador de que esteja ocorrendo uma mobilização de recursos atencionais (CARRETIÉ et al., 2004).

\begin{tabular}{l|l} 
Klados et al. & 32 estudantes \\
universitários
\end{tabular}

\begin{tabular}{|c|c|c|}
\hline & & $\begin{array}{l}\text { problemas aritméticos } \\
\text { (adição e multiplicação de } \\
\text { um e dois dígitos) e uma } \\
\text { tarefa de memória de } \\
\text { trabalho composta por três } \\
\text { níveis de dificuldade, } \\
\text { enquanto } \\
\text { monitorados por medidas } \\
\text { de reatividade } \\
\text { eletrofisiológica- } \\
\text { (Potenciais de Eventos } \\
\text { Relacionados) }\end{array}$ \\
\hline $\begin{array}{l}\text { Pletzer et al. } \\
(2015)\end{array}$ & $\begin{array}{c}36 \\
\text { participantes } \\
\text { adultos }\end{array}$ & $\begin{array}{l}\text { Os participantes realizaram } \\
\text { tarefas não numéricas } \\
\text { (rotação mental e } \\
\text { raciocínio verbal), tarefas } \\
\text { de comparação de números } \\
\text { e tarefas de bissecção de } \\
\text { números }{ }^{6} \text {, enquanto eram } \\
\text { monitorados por RMf. }\end{array}$ \\
\hline
\end{tabular}

Foi notada uma amplitude reduzida de ERP nos locais fronto central e centro parietal dos indivíduos ansiosos durante os estágios iniciais de processamento da tarefa.
Observou-se um aumento na desativação da Rede de Modo Padrão ${ }^{7}$ (RMP), relacionado ao controle atencional. erros na resolução da tarefa de verificação em relação a seus homólogos com baixa ansiedade. Notou-se também que soluções incorretas foram mais plausíveis para o grupo de alta ansiedade, em comparação com seus pares menos ansiosos. Este achado está em acordo com a Teoria do Controle Atencional de Eysenck, mostrando que a AM impactou a eficácia do desempenho (nível de desempenho) e a eficiência de processamento (tempo para processar a tarefa).

As evidências confirmaram que as diferenças significativas entre indivíduos com alta e baixa AM estão localizadas principalmente no córtex préfrontal. Sabe-se que o córtex frontal está associado à Memória de Trabalho e na organização de objetivos cognitivos (MIYAKE et al., 2000; FUSTER, 2002). Os dados informam ainda que os efeitos da AM estão associados a menor ativação cortical durante estágios iniciais do processamento de estímulos numéricos no contexto de tarefas cognitivas.

A AM prejudica a desativação da RMP durante tarefas matemáticas, indicando uma redução na eficiência de processamento. Os indivíduos com alta AM precisam de maior esforço para controlar respostas emocionais negativas para alcançar um desempenho comparável aos indivíduos com baixa ansiedade. Esse

\footnotetext{
${ }^{6} \mathrm{Na}$ tarefa de bissecção de números, os participantes tiveram que decidir se, dados três números inteiros, o número do meio era a média dos outros dois adjacentes (por exemplo: 12_15_18). No exemplo dado, o 15 seria a média entre a soma de 12 com 18.

7 A rede de modo padrão se refere a grupos de neurônios de diferentes regiões encefálicas interconectados que têm ativação elevada em períodos de repouso, mesmo na ausência de qualquer tarefa; por outro lado, tem atividade diminuída em condições ativas.
} 


\begin{abstract}
Klados et al. (2017)

33 estudantes universitários

Os estudantes ficaram em repouso antes de serem apresentados a quatro tipos de problemas aritméticos (adição e multiplicação de um e dois dígitos) e uma tarefa de memória de trabalho composta por três níveis de dificuldade. Eles estavam cientes das tarefas. A atividade cerebral durante o repouso foi mensurada por EEG multicanal.
\end{abstract}

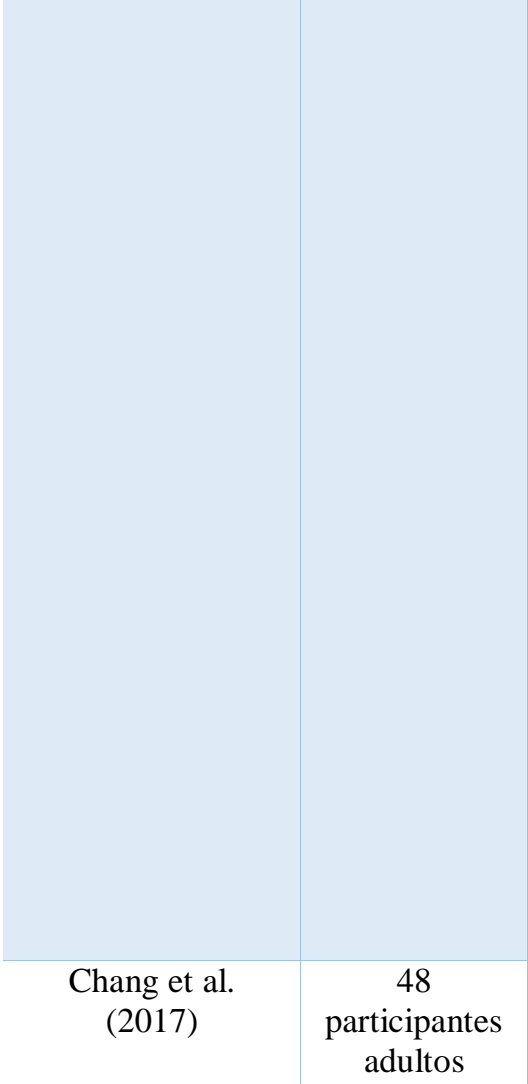

Foi observada uma atividade mais intensa nas bandas de EGG (Alpha, Beta, Gama, Delta e Theta) dos indivíduos com alta AM.

A rede de atenção frontoparietal (giro frontal inferior e lóbulo parietal superior) foi associada ao desempenho de indivíduos com AM. resultado corrobora com o paradigma da Teoria do Controle Atencional, proposta por Eysenck e Calvo (2007).

As evidências sustentam mudanças na rede cerebral de indivíduos com alta AM durante a fase de antecipação da tarefa matemática, o que pode ser interpretado como um sinal de organização mais intensa da rede cerebral desses indivíduos. Segundo os autores, a ativação da banda delta pode ser resultado da inibição de outros processos que interferem na resolução de tarefas matemáticas. A conectividade da banda theta pode ser explicada como uma forte assinatura emocional advinda da expectativa de participar de um programa relacionado à matemática. As bandas Beta e Gama podem estar relacionadas à rede de dor ativada, como mostrado por Lyons e Beilock (2012b), em estudo anterior.

Os indivíduos com alta AM quando comparados com seus pares menos ansiosos, tiveram melhor desempenho quando ativaram menos a rede de atenção frontoparietal. Isso quer dizer que alguns indivíduos ansiosos à Matemática podem recrutar recursos de controle cognitivo para regular respostas emocionais negativas associadas às situações matemáticas, mesmo quando a tarefa de Matemática não for necessariamente complexa. Da mesma forma, a ativação dessa rede pode ser reflexo de um esforço aumentado ou eficiência diminuída ao processar informações numéricas. As evidências também mostram que indivíduos com alta AM 


\begin{tabular}{|c|c|c|c|c|}
\hline & & & & $\begin{array}{l}\text { podem abordar os problemas } \\
\text { matemáticos de maneira } \\
\text { diferente dos indivíduos com } \\
\text { baixa AM. De modo geral, } \\
\text { estudantes que experimentam } \\
\text { altos níveis de AM podem se } \\
\text { beneficiar de práticas de sala } \\
\text { de aula que ajudem os alunos } \\
\text { a regular suas emoções } \\
\text { negativas e a desenvolver } \\
\text { fluência na recuperação de } \\
\text { fatos aritméticos simples. }\end{array}$ \\
\hline $\begin{array}{l}\text { Pizzie e Kraemer } \\
\qquad(2017)\end{array}$ & $\begin{array}{l}40 \text { estudantes } \\
\text { universitários }\end{array}$ & $\begin{array}{lr}\text { Os participantes são } \\
\text { expostos a } & \text { imagens } \\
\text { envolvendo } & \text { símbolos } \\
\text { neutros e } & \text { símbolos } \\
\text { matemáticos, enquanto são } \\
\text { monitorados por fMRI. }\end{array}$ & 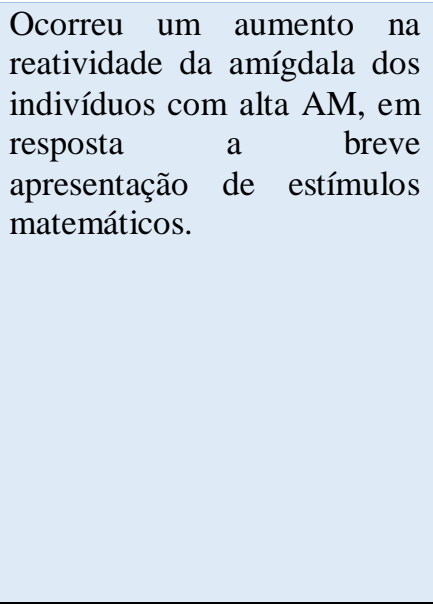 & $\begin{array}{l}\text { A ativação da amígdala após } \\
\text { apresentação de estímulos } \\
\text { matemáticos vem ilustrar que } \\
\text { indivíduos com alta AM } \\
\text { apresentam uma assinatura } \\
\text { neural associada à ameaça e } \\
\text { vigilância, mesmo quando } \\
\text { não precisam antecipar ou se } \\
\text { esforçar para resolver } \\
\text { problemas matemáticos } \\
\text { difíceis. Esses resultados } \\
\text { corroboram as evidências de } \\
\text { Young et al. (2012), } \\
\text { ampliando para indivíduos } \\
\text { jovens. }\end{array}$ \\
\hline $\begin{array}{l}\text { Kucian et al. } \\
\text { (2018) }\end{array}$ & $\begin{array}{l}63 \text { crianças } \\
\text { entre } 7-16 \\
\text { anos }\end{array}$ & $\begin{array}{l}\text { Todas as crianças foram } \\
\text { submetidas a testes } \\
\text { neuropsicológicos } \\
\text { detalhados, incluindo } \\
\text { desempenho matemático, } \\
\text { memória de trabalho, } \\
\text { dentre outros, enquanto } \\
\text { eram monitorados por } \\
\text { ressonância magnética. }\end{array}$ & $\begin{array}{l}\text { Notou-se uma diminuição no } \\
\text { volume da amígdala direita } \\
\text { em crianças com alta AM. }\end{array}$ & $\begin{array}{l}\text { A AM não somente dificulta } \\
\text { o desenvolvimento aritmético } \\
\text { em crianças, como também } \\
\text { está relacionada a estrutura } \\
\text { cerebral alterada de áreas } \\
\text { responsáveis por processar o } \\
\text { medo. }\end{array}$ \\
\hline
\end{tabular}

Quadro 2 - Literatura Neurocientífica sobre os correlatos Neurais da Ansiedade Matemática. Os estudos estão organizados pelo nome de seus pesquisadores, pelo tamanho de suas populações, pelos procedimentos realizados, por seus correlatos neurais e por suas implicações.

Fonte: Pubmed (National Library of Medicine National Institutes of Health dos EUA) e ERIC (Education Resources Information Center)

No Quadro 2, analisamos pormenorizadamente os 14 estudos incluídos em nossa Revisão Sistemática, destacando suas amostras, procedimentos, correlatos neurais associados à AM e algumas de suas implicações.

\section{Discussão}

Os poucos estudos conduzidos por fMRI mostraram que, pelo menos duas bases, cerebrais estão envolvidas na resposta emocional da Ansiedade Matemática: a rede de medo e a rede de 
dor. Sugere-se que os déficits de desempenho matemático em indivíduos com alta AM estão associados a atividades nessas regiões cerebrais, comumente envolvidas na regulação de emoções negativas, assim como em regiões subcorticais relacionadas a fatores motivacionais (LYONS; BEILOCK, 2012a ARTEMENKO et al., 2015). Esses estudos sugerem que tais indivíduos podem superar seus déficits de desempenho aprendendo a controlar essa resposta emocional negativa, mesmo que a manipulação numérica envolva custos significativos de memória de trabalho.

Notou-se ainda que, para a maioria dos indivíduos com alta AM, estímulos aversivos (isto é, os números) contribuem para reduzir a capacidade de recursos na memória de trabalho, que poderiam ser alocados para a tarefa cognitiva. Como os números estão vinculados à própria tarefa matemática em si, pode-se supor que esses indivíduos, ao se preocuparem com o conteúdo emocional dos estímulos, reduzem recursos na Memória de Trabalho, prejudicando o desempenho, principalmente em tarefas de alta carga cognitiva.

Déficits na função inibitória também foram considerados. As áreas cerebrais relacionadas ao controle atencional (por exemplo, o Córtex Pré-Frontal Dorsolateral e a Rede de Modo padrão) tem sido associadas à eficiência de processamento (FALES et al., 2008 ). Quanto maior a ativação nessas regiões, maior a resposta de controle atencional. Notou-se, entretanto, que indivíduos com alta AM tiveram atividades reduzidas no córtex pré-frontal dorsolateral (YOUNG et al., 2012; PLETZER et al., 2015 ) e lentidão para a desativação da Rede de Modo Padrão (PLETZER et al., 2015). Conforme Anticevic et al. (2010) e Sambataro et al. (2010), a diminuição de atividades em áreas da Rede de Modo Padrão indica um aumento na ativação de áreas de controle cognitivo (uma vez que elas são inversamente proporcionais). Por conta disso, percebe-se que indivíduos com alta AM apresentam um controle atencional insuficiente, afetando, de modo particular, tarefas que requerem funções inibitórias e controle atencional.

\section{Conclusão e implicações para o processo de ensino e aprendizagem}

O corpo de evidências analisadas trás implicações importantes para a prática do professor que ensina Matemática e para o processo de ensino e aprendizagem matemático, de um modo geral. Conforme sugerido em Lyons e Beilock (2012a), a competência matemática de indivíduos 
com alta AM está mais relacionada a organização de recursos de controle cognitivo do que na promoção de intervenções que foquem meramente em treino adicional de Matemática.

Considerando que indivíduos com alta ansiedade matemática são mais propensos a autogerar erros em tarefas numéricas (SUARÉZ-PELLICIONI, 2013a; NÚNES-PEÑA; SUÁREZ-PELLICIONI, 2015) e ponderando que os erros são vistos de modo negativo em aulas de Matemática, provavelmente ele constitui fator contributivo no desenvolvimento e manutenção da Ansiedade Matemática. Intervenções educativas que redimensionem positivamente o "erro" em tarefas matemáticas podem ter implicações positivas no processo de ensino e aprendizagem e pode vir a quebrar o ciclo viciante da Ansiedade Matemática que gera mau desempenho, que, por sua vez, gera mais Ansiedade Matemática.

As evidências destacam ainda que os efeitos com AM estão associados a menor ativação cortical durante estágios iniciais do processamento de estímulos numéricos no contexto de tarefas cognitivas, independente da complexidade da tarefa (KLADOS, 2017). Anteriormente a isso, a AM prejudica a desativação da RMP (Rede de Modo Padrão) durante tarefas matemáticas, indicando uma redução na eficiência de processamento. Pautados nessas perspectivas, hipotetizamos que práticas pedagógicas que considerem a característica reativa dos alunos com AM e que promovam, de modo gradual, uma atitude proativa perante problemas matemáticos, podem beneficiar esses indivíduos a médio e longo prazo.

Ademais, considerando que os indivíduos com alta AM precisam de maior esforço para controlar respostas emocionais negativas para alcançar um desempenho comparável aos indivíduos com baixa ansiedade, hipotetiza-se que atividades que envolvam interação entre os ansiosos e seus homólogos não ansiosos, podem não surtir efeitos positivos.

Os indivíduos com alta AM quando comparados com seus pares menos ansiosos, tiveram melhor desempenho quando ativaram menos a rede de atenção frontoparietal. Isso quer dizer que alguns indivíduos ansiosos à Matemática podem recrutar recursos de controle cognitivo para regular respostas emocionais negativas associadas às situações matemáticas, mesmo quando a tarefa de Matemática não for necessariamente complexa. Da mesma forma, a ativação dessa rede pode ser reflexo de um esforço aumentado ou eficiência diminuída ao processar informações numéricas. As evidências também mostram que indivíduos com alta AM podem abordar os problemas matemáticos de maneira diferente dos indivíduos com baixa AM. De um modo geral, estudantes que experimentam altos níveis de AM podem se beneficiar de práticas de sala de aula 
que ajudem os alunos a regular suas emoções negativas e a desenvolver fluência na recuperação de fatos aritméticos simples.

Nosso estudo procurou estabelecer um movimento translacional, sistematizando os trabalhos publicados na última década acerca das bases neurais da Ansiedade Matemática, evidenciando como tais achados podem influenciar as ações do professor que ensina Matemática na sala de aula. Dentre algumas de nossas limitações metodológicas, destacamos as poucas bases de dados consideradas e o recorte temporal dos últimos 10 anos, que, em ocasiões futuras, poderão ser revistas e ampliadas. Destacamos, entretanto, os pontos fortes de nossa revisão, como o uso do diagrama PRISMA que identificou, triou, elegeu e incluiu os estudos pesquisados e a Escala de Qualidade PEDro que avaliou os estudos incluídos, propiciando para a área de Educação Matemática, resultados recentes em neurociência cognitiva para discutir um transtorno de aprendizagem conhecido nas aulas de Matemática.

\section{Referências}

ANTICEVIC, A.; REPOVS, G.; SHULMAN, G.L., BARCH, D. M. When less is more: TPJ and default network deactivation during encoding predicts working memory performance. Neuroimage, v. 49, n.3, p. 2638-2648, feb. 2010.

ASHCRAFT, M. H.; FAUST, M. W. Mathematics anxiety and mental arithmetic performance: An exploratory investigation. Cognition and Emotion, Cleveland State University, EUA, v. 8, n. 2, p. 97-125, mar.1994.

ASHCRAFT, M. H.; KIRK, E. P. The relationships among working memory, math anxiety, and performance. Journal of Experimental Psychology: General, Washington, v. 130, n. 2, p. 224-237, jul. 2001.

ASHCRAFT, M. H. Math anxiety: Personal, educational, and cognitive consequences. Current Directions in Psychological Science, Cleveland, Ohio, v. 11, n. 5, p. 181-185, Oct. 2002.

ASHCRAFT, M. H.; RIDLEY, K. Math anxiety and its cognitive consequences: A tutorial review. In: CAMPBELL, J. I. D. (Ed.). Handbook of mathematical cognition. New York: Psychology Press, 2005. p. 315-325.

ASHCRAFT, M. H.; MOORE, A. M. Mathematics Anxiety and the Affective Drop in Performance. Journal of Psycho educational Assessment, Las Vegas, v. 27, n. 3, p. 197-205, jun. 2009.

ARTEMENKO, C.; DAROCZY, G.; NUERK H. C. Neural correlates of math anxiety - an overview and implications. Frontiers in Psychology, Cambridge, v. 6, p. 1333. sep. 2015.

BEILOCK, S. L.; DECARO, M. S. From Poor Performance to Success under Stress: Working 
Memory, Strategy Selection, and Mathematical Problem Solving Under Pressure. Journal of Experimental Psychology: Learning, Memory, and Cognition, Washington, v. 33, n. 6, p. 983-998, nov. 2007.

BRASS, M.; DERRFUSS, J.; FORSTMANN, B.; VON CRAMON, D. Y. The role of the inferior frontal junction area in cognitive control. TRENDS in Cognitive Science, Canadá v. 9, n.7, p. 314-316, jul. 2005.

BROWN, M.; BROWN, P.; BIBBY, T. "I would rather die": reasons given by 16 -year-olds for not continuing their study of mathematics. Research in Mathematics Education, London, v. 10, n.1, p. 3-18, sep. 2008.

CAREY, E.; HILL, F.; DEVINE, A.; SZÜCS, D. The Chicken or the Egg? The Direction of the Relationship between Mathematics Anxiety and Mathematics Performance. Frontiers in Psychology, Belgium, v. 6, n. 33, jan. 2016.

CARRETIÉ, L., et al. Automatic attention to emotional stimuli: Neural correlates. Human Brain Mapping, United States, v. 22, n. 4, p. 290-299. 2004.

CHANG, C.; CROTTAZ-HERBETTE, S.; MENON, V. Temporal dynamics of basal ganglia response and connectivity during verbal working memory. Neuroimaging, v. 34, n.3, p. 1253-1269, feb. 2007.

CHANG, H.; SPRUTE, L.; MALONEY, E. A.; BEILOCK, S. L.; BERMAN, M. G. Simple arithmetic: not so simple for highly math anxious individuals. Social Cognitive and Affective Neuroscience, Oxford University Press v. 12, n. 12, p. 1940-1949, dec. 2017.

DERRFUSS, J.; BRASS, M.; NEUMANN, J.; VON CRAMON, D.Y. Involvement of the inferior frontal junction in cognitive control: meta-analyses of switching and Stroop studies. Human Brain Mapping, v. 25, n. 1, p. 22-34, may. 2005.

DERRFUSS, J.; BRASS, M.; VON CRAMON, D.Y.; LOHMANN, G.; AMUNTS, K. Neural activations at the junction of the inferior frontal sulcus and the inferior precentral sulcus: interindividual variability, reliability, and association with sulcal morphology. Human Brain Mapping, v. 30, n. 1, p. 299 - 311, jan. 2009.

DREGER, R. M.; AIKEN Jr., L. R. The identification of number anxiety in a college population. Journal of Educational Psychology, Washington, v. 48, n. 6, p. 344-351, 1957.

DOWKER, A.; SARKAR, A.; LOOI, C. Y. Mathematics Anxiety: What Have We Learned in 60 Years? Frontiers in Psychology, Washington,v. 25, n. 7 p. 508, apr. 2016.

EYSENCK, M. W.; CALVO, M. G. Anxiety and Cognitive Performance: Attentional Control Theory. Emotion, Washington, v. 7, n. 2, p. 336-353, may. 2007.

FALES, C. L.; et al. Altered emotional interference processing in affective and cognitive control brain circuitry in major depression. Biological Psychiatry, Netherlands, v. 63, n. 4, p. 377- 384, feb. 2008.

FUSTER, J. M. Frontal lobe and cognitive development. Journal of Neurocytology, v. 31, n. 3-5, p. 373385, mar. /jun. 2002. 
HLALELE, D. Exploring rural high school learners' experience of mathematics anxiety in academic settings. South African Journal of Education, South Africa, v. 32, n. 3, p. 268-278, aug. 2012

HEMBREE, R. The nature, effects, and relief of mathematics anxiety. Journal for Research in Mathematics Education, v. 21, n.1, p. 33-46, jan. 1990

JANSEN, B. R. J.; LOUWERSE, J.; STRAATEMEIER, M.; VAN DER VEN, S. H. G.;

KLINKENBERG, S.; VAN DER MAAS, H. L. J. The influence of experiencing success in math on math anxiety, perceived math competence, and math performance. Learning and Individual Differences, United Kingdom, v. 24, p. 190-197, apr. 2013.

JONES, W. J; CHILDERS, T. L; JIANG, Y. The shopping brain: Math anxiety modulates brain responses to buying decisions, Biological Psychology, v. 89, n. 1, p. 201-213, jan. 2012.

KLADOS, M. A.; SIMOS, P.; MICHELOYANNIS, S.; MARGULIES, D.; BAMIDIS, P. D. ERP measures of math anxiety: how math anxiety affects working memory and mental calculation tasks? Frontiers in Behavioral Neuroscience, Braga, Portugal. v. 9, p. 1-9 oct. 2015.

KLADOS, M. A; PANDRIA, N.; MICHELOYANNIS, S.; MARGULIES, D.; PANAGIOTIS, D. Math anxiety: Brain cortical network changes in anticipation of doing mathematics. International Journal of Psychophysiology, v. 122, p. 24-31, dec. 2017.

KUCIAN, K; MCCASKEY, U.; O'GORMAN TUURA, R.; VON ASTER, M. Neurostructural correlate of math anxiety in the brain of children. Translational Psychiatry, United Kingdom, v. 8, n. 273, p. 2-11, dec. 2018.

LAMM, C.; DECETY, J.; SINGER, T. Meta-analytic evidence for common and distinct neural networks associated with directly experienced pain and empathy for pain. NeuroImage, United States , v. 54, n. 3, p. 2492-2502, feb. 2011.

LEDOUX, J. E., \& PHELPS, E. A. Emotional networks in the brain. In: Lewis, J. M. Haviland-Jones, \& L. F. Barrett (Eds.), Handbook of emotions, United States, p. 159-179, 2008.

LYONS, I. M.; BEILOCK, S. L. Mathematics Anxiety: Separating the Math from the Anxiety.

Cerebral Cortex, United Kingdom, v. 22, n. 9, p. 2102- 2110, sep. 2012a.

LYONS, I. M.; BEILOCK, S. L. When Math Hurts: Math Anxiety Predicts Pain Network Activation in Anticipation of Doing Math. PLoS ONE, United State, v. 7, n. 10, p.1-6, oct. 2012 b.

MENON, V. Developmental cognitive neuroscience of arithmetic: implications for learning and education. ZDM Germany, v. 42, n. 6, p. 515-525, oct. 2010.

MENON, V.; RIVERA, S. M.; WHITE, C. D.; GLOVER, G. H.; REISS, A. L. Dissociating prefrontal and parietal cortex activation during arithmetic processing. NeuroImage, United States, v. 12, n. 4, p. 357365 , oct. 2000.

MIYAKE, A.; FRIEDMAN, N. P.; EMERSON, M. J.; WITZKI, A. H.; HOWERTER, A. The Unity and Diversity of Executive Functions and Their Contributions to Complex "Frontal Lobe" Tasks: A Latent Variable Analysis. Cognitive Psychology, United States, v. 41, n. 1, p. 49-100, aug. 2000. 
MOHER, D.; LIBERATI, A.; TETZLAFF, J.; ALTMAN, D.G. The PRISMA Group. Preferred Reporting Items for Systematic Reviews and Meta-Analyses: The PRISMA Statement. PLoS Medicine, United States, v. 6, n. 7, p.332-336, jul. 2009.

MUTODI, P.; NGIRANDE, H. Exploring Mathematics Anxiety: Mathematics Students' Experiences. Mediterranean Journal of Social Sciences, Italy, v. 5, n. 1, p.283-294, 2014.

NÚNEZ-PEÑA, M. I.; SUÁREZ-PELLICIONI, M. Less precise representation of numerical magnitude in high math-anxious individuals: An ERP study of the sizeand distance effects. Biological Psychology, Netherlands, v. 103, p. 176-183, dec. 2014.

NÚÑEZ-PEÑA, M. I.; SUÁREZ-PELLICIONI, M. Processing of multi-digit additions in high math anxious individuals: psychophysiological evidence. Frontiers in Psychology, Switzerland, v. 6:, p.1-11, aug. 2015.

OECD, PISA 2012 Results: Ready to Learn: Students' Engagement, Drive and Self-Beliefs (Volume III), PISA, OECD Publishing, 2012.

PESSOA, L. On the relationship between emotion and cognition. Nature Reviews Neuroscience, United Kingdom, v. 9, n. 2, p. 148-158, feb. 2008.

PHELPS E. A; LEDOUX, J. E. Contributions of the amygdala to emotion processing: from animal models to human behavior. Neuron, United States v. 48, n. 2, p. 175-187, oct. 2005.

PLETZER, B.; KRONBICHLER, M.; NUERK, H-C.; KERSCHBAUM, H. H. Mathematics anxiety reduces default mode network deactivation in response to numerical tasks. Frontiers in Human Neuroscience, Switzerland, v. 9, p.1-12, apr. 2015.

PIZZIE, R. G.; KRAEMER, D. J. M. Avoiding math on a rapid timescale: Emotional responsivity and anxious attention in math anxiety. Brain and Cognition, United States, v. 118, p. 100-107, nov. 2017.

RICHARDSON, F. C.; SUINN, R. M. The Mathematics Anxiety Rating Scale: Psychometric data. . Journal of Counseling Psychology, United States, v. 19, n. 6, p. 551-554, 1972.

SAMBATARO, F. et al. Age related alterations in default mode network: impact on working memory performance. Neurobiology of Aging, Netherlands, v. 31, n. 5, p. 839-852, may. 2010.

SARKAR, A.; DOWKER, A.; COHEN KADOSH, R. Cognitive Enhancement or Cognitive Cost: Trait Specific Outcomes of Brain Stimulation in the Case of Mathematics Anxiety. The Journal of Neuroscience, United States, v. 34, n. 50, p. 16605-16610, dec. 2014.

SHIWA, S. R.; PENA COSTA, L. O.; MOSER, A. D. L. Pedro: a base de dados de evidências em fisioterapia. Fisioterapia em Movimento, Curitiba, v. 24, n. 3, p. 523-533, jul. /set. 2011.

SUÁREZ-PELLICIONI, M.; NÚÑEZ-PEÑA, M. I.; COLOMÉ, À. Abnormal Error Monitoring in MathAnxious Individuals: Evidence from Error-Related Brain Potentials. PLoS ONE, United States, v. 8, n. 11, p.1-17, nov. 2013a.

SUÁREZ-PELLICIONI, M.; NÚNEZ-PEÑA, M. I.; COLOMÉ, A. Mathematical anxiety effects on simple arithmetic processing efficiency: An event-related potential study. Biological Psychology, Netherlands, v. 94, n. 3, p. 517-526, dec. 2013 b. 
SUÁREZ-PELLICIONI, M.; NUNEZ-PENA, M. I.; COLOMÉ, A. Reactive Recruitment of Attentional Control in Math Anxiety: An ERP Study of Numeric Conflict Monitoring and Adaptation. PLoS ONE, United States, v. 9, n. 6, p.1-15, jun. 2014.

SUÁREZ-PELLICIONI, M; NÚÑEZ-PEÑA, M. I.; COLOMÉ, À. Math anxiety: A review of its cognitive consequences, psychophysiological correlates, and brain bases. Cognitive, Affective and Behavioral Neuroscience, United States, v. 16, n. 1, p. 3-22, feb. 2016.

SUPEKAR, K.; IUCULANO, T.; CHEN, L.; MENON, V. Remediation of Childhood Math Anxiety and Associated Neural Circuits through Cognitive Tutoring. The Journal of Neuroscience, United States, v. 35, n. 36, p. $12574-12583$, sep. 2015.

YOUNG, C. B.; WU, S. S.; MENON, V. The Neurodevelopmental Basis of Math Anxiety. Psychological Science, United States, v. 23, n. 5, p. 492-501, mar. 2012.

WENDELKEN, C. Meta-analysis: how does posterior parietal cortex contribute to reasoning? Frontiersin Human Neuroscience., Switzerland, v. 8, p.1-11, jan. 2015.

Submetido em 12 de Setembro de 2018 Aprovado em 04 de Novembro de 2019. 